\title{
A Pilot Study of the Creighton Model System of Natural Family Planning in a Subpopulation of Women in Enugu, South-East Nigeria
}

\author{
Ijeoma Ezeome ${ }^{*}$, Margaret Iloanusi ${ }^{2}$, Samuel Obi $^{1}$
}

\begin{abstract}
Objectives: Sixteen percent of Nigerian women have an unmet need for family planning (FP) as a result of the fear of contraceptive side effects and religious beliefs. Increasing the range of accessible contraceptives, especially addition of fertility awareness options is likely to reduce unmet need. This study sought to assess the factors influencing the use of the Creighton model system (CrMS) of natural family planning (NFP) and its effectiveness in a subpopulation of women in Enugu, Southeast Nigeria.

Materials and Methods: This was a cross-sectional descriptive study involving all consenting women attending the family planning clinic (FPC) of a private hospital, through a structured questionnaire and information from follow-up (FU) form of each consenting client. Data analysis was done using SPSS version 17.0 and results were expressed in descriptive statistics of frequency and percentage. Results: Forty-five couples consented to the study. The age range of the women was $22-47$ years, with a mean of 32 years. Forty-seven percent of the women had a tertiary education. Two-thirds (67\%) of the couples were using the system to temporarily space their pregnancies and $60 \%$ of them were Roman Catholics. One "unplanned" pregnancy occurred during the period of review (October 2010-October 2016). The major reason for discontinuance was the tension created between the spouses during periods of avoiding pregnancy. The major advantage was being rid of the fear of side effects of artificial contraceptive methods.

Conclusions: Roman Catholicism and high motivation were factors influencing the use of this FP model. For couples who can learn the method, the CrMS of FP is an effective option for regulating family size.

Keywords: Fertility awareness-based methods, Family planning, Creighton model system
\end{abstract}

\section{Introduction}

With a total population of 175 million and an annual growth rate of $3.2 \% .{ }^{1}$ Nigeria remains the most highly populated country in Africa. It accounted for $14 \%$ of the global maternal deaths in $2013 .^{2}$ Limiting or spacing of pregnancies remain one of the most effective means of preventing maternal and child mortality. ${ }^{3}$ Hence providing avenues for increased accessibility and uptake of contraceptives would improve maternal health and thus reduce deaths. ${ }^{4}$

Sixteen percent of married Nigerian women want to avoid pregnancy but are not using a contraceptive method, ${ }^{1}$ while some 222 million women worldwide have unmet needs for contraception. ${ }^{5}$ Increasing supply and access to contraception is likely to reduce this need by encouraging more women to use modern contraception. ${ }^{6}$ The reasons women give for not using contraceptives include healthrelated risks, inconveniences of the method, and opposition to the method by themselves or a close relation. ${ }^{7}$ The contraceptive needs of men and women are not being fully met by the family planning (FP) options presently being promoted. The Nigerian Government aimed to increase access to FP, with a target of $36 \%$ contraceptive prevalence rate (CPR) by $2018 .^{3}$ The key to achieving this goal is by a diverse method mix including fertility awareness based methods (FABMs), considering the multi-religious and multi-ethnic composition of the Nigerian society with different sociocultural norms. The FABMs are not only safe, based on a thorough understanding of reproductive biology but have also undergone rigorous effectiveness studies, hence fulfilled conditions as modern methods for avoiding pregnancy. ${ }^{8}$ Additionally, they are comparatively inexpensive, not under the influence of any religious beliefs, can be practiced secretly by partners ${ }^{9}$ and involve no medical appliance or medicine. ${ }^{5}$

Since it is generally known that the older natural fertility regulation methods such as basal body temperature alone and calendar rhythm have low effectiveness, many women do not know of the availability of modern highly effective FABMs. ${ }^{10}$ However, their effectiveness depends on proper observation and correct recording of the fertility biomarkers as well as following instructions given to them by well-trained fertility care providers. ${ }^{11}$

Current methods of comparing the effectiveness of FABMs and contraceptives are flawed. A method can only be said to have failed despite its perfect use. ${ }^{10}$ 
The Creighton Model is one of the FABMs that involves identifying changes in the cervical mucus, just like the Billings ovulation method (BOM). Its difference lies in the characterization of the secretions with the aid of a picture dictionary (PD) and standardized terminology. ${ }^{12}$ Pregnancy is avoided through abstinence alone. It involves the observation and characterization of the cervical mucus throughout the day, including when one is about to go to bed. Correct identification of the cervical mucus indicates that ovulation is approaching and its presence is of utmost importance for sperm survival. Timed intercourse at this period will thus lead to high chances of pregnancy. ${ }^{13}$

In the literature, there are studies on using the Creighton model system (CrMS) for avoiding pregnancy among women of European and American descent. ${ }^{12,14}$ Though studies of the use of fertility based awareness methods have been carried out in some African countries, ${ }^{9}$ no study has been carried out specifically to investigate the use of CrMS to avoid pregnancy among African women, nor Nigerian women to our knowledge. Hence it is hoped that this pilot study will highlight the factors influencing the use of this natural family planning (NFP) model for avoiding pregnancy as well as assess its effectiveness in this cohort of women.

\section{Materials and Methods}

This is a prospective, descriptive pilot study with a sample size of 90 clients, comprising 45 couples who were seen in the Creighton Model family planning clinic (FPC) of a private specialist hospital in Enugu, Nigeria. Women who attended the antenatal and gynecological clinics of the hospital were made aware of the FPC through talks given by nurses while waiting to consult the doctor. Those who were interested were given appointments for an introductory session (IS) on the FP Model which can be used to either avoid or achieve pregnancy. The services were personalized to the particular client hence run daily throughout the week depending on time agreed on by the client and fertility care practitioners (FCPs). A total of 60 couples were registered in the clinic but 45 couples agreed to take part in the study. The couples started using the CrMS from October 2010. The period of the study spanned 72 months (6 years), up to October 2016. They all filled a standardized demographic form and were told that the information would be used for evaluation and they gave consent. The Model entailed introductory and follow-up (FU) sessions, evaluations and a thorough training program for the instructors.

The IS was a 1-hour slide presentation explaining the anatomy and physiology of the male and female reproductive system as well as how to chart one's cycle. The couples were given a user manual describing not only the reproductive systems but also the observation of biomarkers to read, fertility monitoring stamps, fertility chart showing where to place stamps and area for describing the vulval discharges, and appointments to meet the practitioner for FU 2 weekly for the first 4 FUs, then 5th FU 1 month later, and the remainders were 3 monthly. The program consisted of a minimum of 8 FUs but might be more depending on each couples' circumstance. Each FU lasted approximately 1 hour. The couples began observation and charting of their fertility biomarkers the next day after the IS and were also instructed to read the user manual. ${ }^{15}$

The women checked cervical mucus by wiping the outside of the vagina each time they urinated, including bedtime after bearing down. The mucus was checked for color, stretch, and consistency. The ovulation day which correlated with the peak day was defined as the last day of clear, stretchy (an inch or more), or lubricative mucus.

Each session was standardized through the use of an FU form which acted as a checklist for all essential items needed for effective learning by the client, as well as monitoring of the clients' learning and growth, a PD of terminology showing different types of discharges to which the client can relate, a user manual, and a case management book for the FCP, who taught the clients.

At each FU session, the observation techniques were evaluated; PD, definitions, and fertility charts were reviewed, while the clients are taught in a progressive manner to correctly identify and value their fertility.

Notwithstanding that the method imparted knowledge in a gradual manner from session to session, the couples were considered the user of the method from the day of the IS. Woven into the fabric of the Model is its quality control. The teacher and the teaching sessions were evaluated during the teaching process, usually at the IS, fourth, fifth and sixth FU sessions. The knowledge and understanding of the clients were tested by asking True (T) or False (F) questions in English or vernacular depending on the clients' fluency. These evaluation tests were standardized for different cycle types (regular, long, breastfeeding, infertility). Irrespective of the intention of use by the couple, should pregnancy occur, an evaluation was done within 3 months.

\section{Data Analysis}

Data analysis was done using SPSS version 17.0 for Windows. Frequency tables were used to find the percentage with regards to demographic data and the different variables under study.

\section{Results}

A total of 60 couples were enrolled in the FP clinic at the time of this report. However, only 45 couples agreed to take part in the study. The age range of the women was 2247 years with a mean of 32 years, while their spouses aged 30-55 years with a mean of 41 years. Ninety-five percent of the women had completed at least primary education while $22 \%$ of the men had no formal education. (Table 1) They were all Christians with $60 \%$ being of Roman Catholic denomination (Table 2). 
Table 1. Age Distribution and Educational Level of Respondents

\begin{tabular}{lcc}
\hline & Woman, No. (\%) & Man, No. (\%) \\
\hline Age (y) & $21(46.67)$ & - \\
$21-30$ & $18(40.00)$ & $14(31.11)$ \\
$31-40$ & $6(13.33)$ & $26(57.78)$ \\
$41-50$ & - & $5(11.11)$ \\
$51-60$ & & \\
Educational level & $2(4.44)$ & $10(22.22)$ \\
Non-formal & $10(22.22)$ & $8(17.78)$ \\
Primary & $12(26.67)$ & $15(33.33)$ \\
Secondary & $21(46.67)$ & $12(26.67)$ \\
Tertiary &
\end{tabular}

Table 2. Religion of the Respondents

\begin{tabular}{lc}
\hline Religion & Number (\%) \\
\hline Roman Catholic & $27(60.00)$ \\
Anglican & $14(31.11)$ \\
Pentecostal & $4(8.89)$ \\
Total & $45(100)$ \\
\hline
\end{tabular}

They had a parity of 2 to 6 with a mean parity of 4 . $40 \%$ and $27 \%$ of the respondents were using The BOM and Intrauterine contraceptive device (IUCD) prior to switching to the CrMS. Their reasons for changing to another method were because of excessive menstrual bleeding, fear of translocation of the IUCD, and distrust of effectiveness of the BOM respectively (Table 3 ). The couples used the method for periods of 1 to 6 years. $67 \%$ of the women were using the system to space their pregnancies (Table 4).

None of the couples were lost to FU. However, seven couples discontinued the use of the system primarily due to difficulty in avoiding during the woman's fertile period. The disadvantages of the system were identified as the high degree of motivation and discipline required of the couples, and avoidance of genital contact during the woman's fertile period. $77 \%$ and $84 \%$ of the couples were satisfied and confident of using the system to avoid pregnancy respectively (Table 5).

A total of 27 planned pregnancies occurred during the 6 years under review among the couples using the system to space pregnancy. One was however due to the fact that the couple took a chance during the woman's fertile period, thus changing their intention for use of the system to Achieving. There was no pregnancy among those using the system to limit pregnancy.

\section{Discussion}

In our study, the clients complained of excessive and irregular uterine bleeding due to the use of hormonal contraceptives and IUCD. This is in line with reasons for low utilization of FP services despite wanting to space or limit births. ${ }^{7,16}$

Sixty percent of our clients were Roman Catholics,
Table 3. Family Planning Methods Prior to Creighton Model System

\begin{tabular}{lcc}
\hline Family Planning Method & No. (\%) & Reason for its Stoppage \\
\hline Calendar/withdrawal & $2(4.44)$ & Distrust effectiveness \\
Calendar/condom & $2(4.44)$ & Distrust effectiveness \\
Calendar & $4(8.89)$ & Distrust effectiveness \\
Billings ovulation & $18(40.00)$ & Distrust effectiveness \\
Norplant insertion & $1(2.22)$ & Irregular bleeding \\
Intrauterine device & $12(26.67)$ & Excessive bleed/translocation \\
Progestogen injection & $6(13.33)$ & Irregular bleeding \\
Total & $45(100)$ & \\
\hline
\end{tabular}

Table 4. Use of Creighton Model System

\begin{tabular}{lc}
\hline & No. (\%) \\
\hline Intention for use & $15(33.33)$ \\
Limit (permanently avoid pregnancy) & $30(66.67)$ \\
Space (temporarily avoid pregnancy) & \\
Length of use (mon) & $3(6.67)$ \\
12 & $3(6.67)$ \\
24 & $2(4.44)$ \\
36 & $1(2.22)$ \\
48 & $36(80.00)$ \\
60 & \\
Continuance/discontinuance & $38(84.44)$ \\
Continued use & $7(15.56)$ \\
Discontinued use & \\
Major reasons for discontinuance & 5 \\
Tension between couples during fertility & 1 \\
Difficulty in making 100\% observations & 1 \\
Uncertainty about fertile period & \\
Disadvantages of the system (as many options apply) & \\
Requires high degree of motivation to use sys & $45(100)$ \\
Difficulty avoiding intercourse during fertile & $40(80.89)$ \\
Lack of sexual spontaneity between couples & $8(17.78)$ \\
Observing for mucus all the time & $5(11.11)$ \\
Tension between husband and wife & $10(22.22)$ \\
\hline
\end{tabular}

probably because they were encouraged by the Church to use FABMs. However, religion alone is not responsible for the success of the method. ${ }^{17}$ Research clearly demonstrates that a high degree of motivation is needed to use FABMs effectively. ${ }^{18}$ Eighty-four percent of our clients had a strong conviction in using the method and were satisfied with the method.

None of our clients using the method to limit pregnancy got pregnant, while those using the method for spacing had planned pregnancies, except for a couple who changed their intention of use from avoiding to achieving by using the fertile period for genital intercourse. This goes to support the first effectiveness study of the CrMS which found a method and use-effectiveness of $99.5 \%$ and $96.8 \%$, respectively. ${ }^{12}$ Similar studies also found a method and use-effectiveness of $98.8 \%$ and $98.0 \%$ respectively in avoiding pregnancy, with a continuation rate of $78.0 \%$ after 12 months of use. ${ }^{15}$ The couples were taught at the IS 
Table 5. Conviction, confidence and satisfaction using the Creighton Model System

\begin{tabular}{lc}
\hline & No. (\%) \\
\hline Strength of conviction using system for intent & 0 \\
Definitely not strong & $5(11.11)$ \\
Not strong & $2(4.44)$ \\
Not sure (undecided) & $38(84.45$ \\
Strong & 0 \\
Very strong & \\
Confidence in using System for Intention & 0 \\
Very confident & $5(11.11)$ \\
Not confident & $2(4.44)$ \\
Undecided & $38(84.45)$ \\
Confident & 0 \\
Very Confident & 0 \\
Overconfident & \\
Satisfaction using the system for intention & 0 \\
Very unsatisfied & $7(15.56)$ \\
Unsatisfied & 0 \\
Not sure (undecided) & $35(77.77)$ \\
Satisfied & $3(6.67)$ \\
Very satisfied &
\end{tabular}

and FUs that using a day of fertility for intercourse means that they have changed their use of the method from avoiding to achieving. The success or effectiveness of the method depends on the personal choices of the couple to avoid or have intercourse on the day of fertility and not on whether the pregnancy is desired or not.

The difficulty in avoiding genital contact during the fertile period was given by many of our clients as a major disadvantage to the system, while tension between couples during this period was mentioned as a major reason for discontinuance. We had a discontinuance rate of $15 \%$. Spouses living apart and non-supportive husbands were the factors found to affect the success of FABMs. ${ }^{19,20}$ The rate of use remained low because many women believed that these methods were not only ineffective but cumbersome, and misconceptions of most physicians about the effectiveness of the newer FABMs prevented them from recommending them. The women in our study were taught the system by certified FCPs trained in teaching of the CrMS.

\section{Conclusions}

The above-mentioned clients have been successful in the use of the method because it was their choice, and they have been properly instructed on how to do so. The modern FABMs are wrongly classified as the same as the non-effective traditional methods of natural fertility regulation, hence not usually included in the FP options promoted and available to women. In order to reduce unmet need and increase the CPR, promotion of a mixture of different methods including modern FABMs is imperative. ${ }^{8}$
Limitation of the Study

An important limitation of the study lies in its small sample size. There is a need for it to be replicated with a population of diverse religion, socioeconomic and educational status, early reproductive years, and single women.

\section{Conflict of Interests}

Authors declare that they have no conflict of interests.

\section{Ethical Issues}

Following an in-depth review of the literature on the topic of study, a draft questionnaire was made and presented to a team of experts for content and face validity. The approval of this study was obtained from the Ethics Committee of the University of Nigeria Teaching Hospital, Enugu. The questionnaire was pretested prior to the commencement of the study. An oral informed consent was also obtained from the women prior to being served with the questionnaire, highlighting that the information being sought would be used strictly for research purposes and treated with utmost confidentiality..

\section{Financial Support}

This study is a self-funded research.

\section{References}

1. Nigeria Demographic and Health Survey (NDHS). Abuja, Nigeria; 2013. http://www.statcompiler.com/.

2. Austin A. Unmet contraceptive need among married Nigerian women: an examination of trends and drivers. Contraception. 2015;91(1):31-38. doi:10.1016/j.contraception.2014.10.002

3. Nigeria Family Planning Blueprint (Scale-up Plan). https:// www.healthpolicyproject.com/ns/docs/CIP_Nigeria.pdf. Accessed online March 28, 2017. Published October 2014.

4. Geleta D, Birhanu Z, Kaufman M, Temesgen R. Gender Norms and Family Planning Decision-Making Among Married Men and Women, Rural Ethiopia: A Qualitative Study. Science Journal of Public Health. 2015;3(2):242-250.

5. Simmons RG, Shattuck DC, Jennings VH. Assessing the Efficacy of an App-Based Method of Family Planning: The Dot Study Protocol. JMIR Res Protoc. 2017;6(1):e5. doi:10.2196/resprot.6886

6. Mukasa B, Ali M, Farron M, Van de Weerdt R. Contraception supply chain challenges: a review of evidence from low- and middle-income countries. Eur J Contracept Reprod Health Care. 2017;22(5):384-390. doi:10.1080/13625187.2017.139 4453

7. Sedgh G, Ashford LS, Hussain R. Unmet need for contraception: examining women's reasons for not using a method. New York: Guttmacher Institute; 2016.

8. Malarcher S, Spieler J, Fabic MS, Jordan S, Starbird EH, Kenon C. Fertility Awareness Methods: Distinctive Modern Contraceptives. Glob Health Sci Pract. 2016;4(1):13-15. doi:10.9745/ghsp-d-15-00297

9. Kabong RM, Baboo KS, Mweemba O. Factors influencing utilization of Natural Family Planning among Child Bearing Women in Chilonga Northern Province Zambia. 
Med J Zambia. 2010;37(4):223-233.

10. Pallone SR, Bergus GR. Fertility awareness-based methods: another option for family planning. J Am Board Fam Med. 2009;22(2):147-157. doi:10.3122/jabfm.2009.02.080038

11. Duane M, Contreras A, Jensen ET, White A. The Performance of Fertility Awareness-based Method Apps Marketed to Avoid Pregnancy. J Am Board Fam Med. 2016;29(4):508-511. doi:10.3122/jabfm.2016.04.160022

12. Hilgers TW, Stanford JB. Creighton Model NaProEducation Technology for avoiding pregnancy. Use effectiveness. J Reprod Med. 1998;43(6):495-502.

13. Stanford JB, Thurman PB, Lemaire JC. Physicians' knowledge and practices regarding natural family planning. Obstet Gynecol. 1999;94(5 Pt 1):672-678.

14. Frank-Herrmann P, Heil J, Gnoth C, et al. The effectiveness of a fertility awareness based method to avoid pregnancy in relation to a couple's sexual behaviour during the fertile time: a prospective longitudinal study. Hum Reprod. 2007;22(5):1310-1319. doi:10.1093/humrep/dem003

15. Fehring RJ, Lawrence D, Philpot C. Use effectiveness of the Creighton model ovulation method of natural family planning. J Obstet Gynecol Neonatal Nurs. 1994;23(4):303309.

16. Sedgh G, Hussain R. Reasons for contraceptive nonuse among women having unmet need for contraception in developing countries. Stud Fam Plann. 2014;45(2):151-169. doi:10.1111/j.1728-4465.2014.00382.x

17. Wilson MA. The practice of natural family planning versus the use of artificial birth control: family, sexual and moral issues; 2002. http://www.lifeissues.net/writers/wils/ wils_01naturalfamilyplanning1.html.

18. Pyper CM, Knight J. Fertility Awareness Methods of Family Planning for Achieving or Avoiding Pregnancy. The Global Library Of Women's Medicine. 2008. doi:10.3843/ GLOWN.10384

19. John NA, Babalola S, Chipeta E. Sexual Pleasure, Partner Dynamics and Contraceptive Use in Malawi. Int Perspect Sex Reprod Health. 2015;41(2):99-107. doi:10.1363/4109915

20. Ali MM, Cleland J, Shah IH. Causes and Consequences of Contraceptive Discontinuation: Evidence from 60 Demographic Health Surveys. Geneva: WHO; 2012.

(c) 2018 The Author (s); This is an open-access article distributed under the terms of the Creative Commons Attribution License (http://creativecommons.org/licenses/by/4.0), which permits unrestricted use, distribution, and reproduction in any medium, provided the original work is properly cited. 